91

دراسة اقتصادية لاور المصرف الزراعي والريفي في تمويل الإنفاق التتموي الزراعي في ليبيا

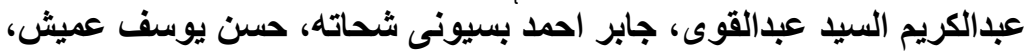

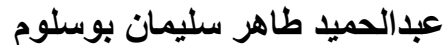

قسم الإرشاد الزراعي والتنمية الريفية، كلية الزراعة، جامعة عمر المختار ـ ليبيا.

مستخلص

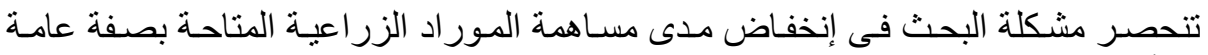

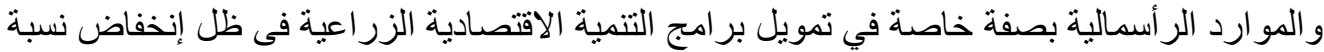

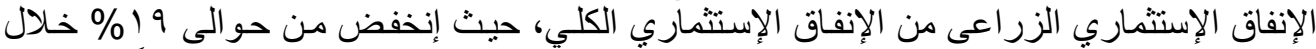

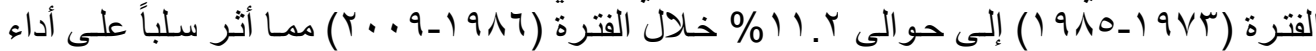

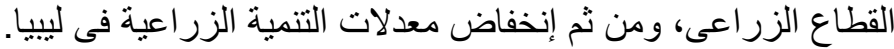

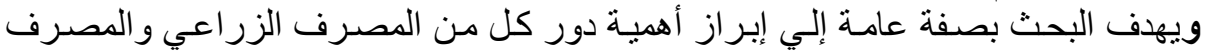

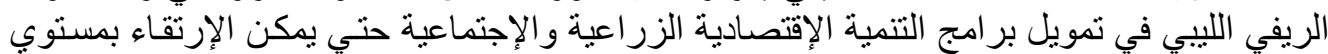

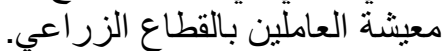

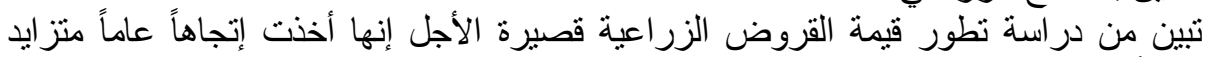

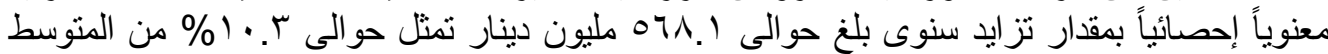

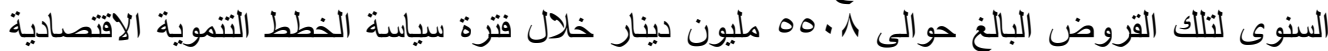

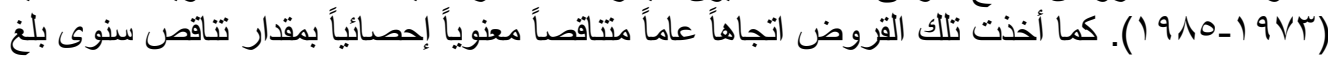

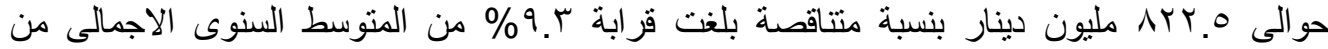

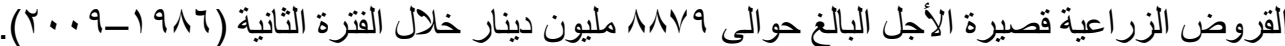

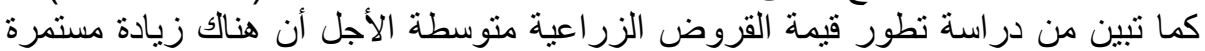

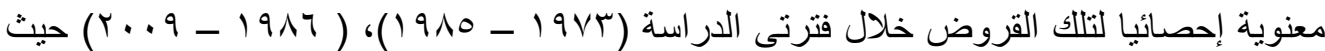

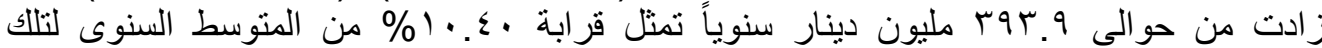

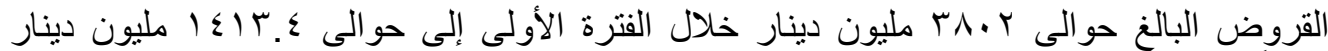

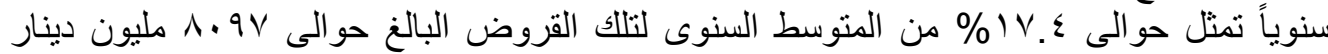

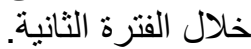

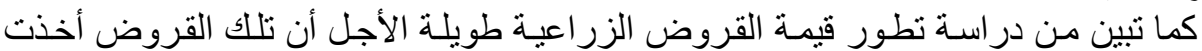

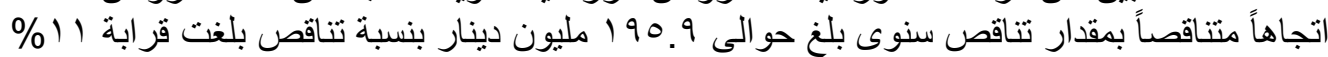

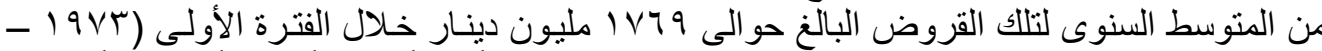

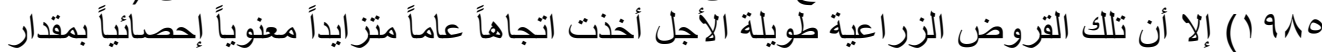

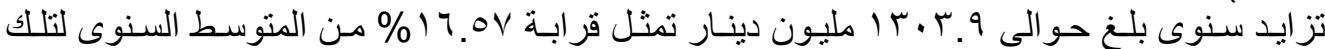

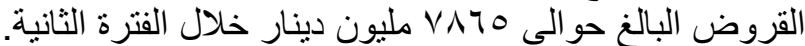

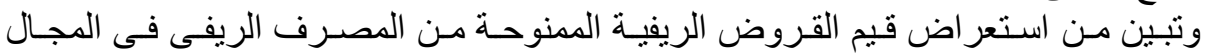

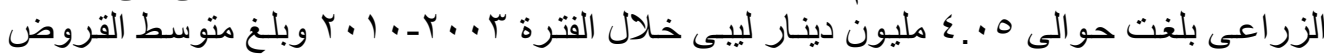

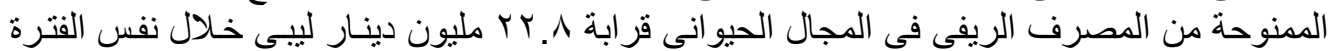

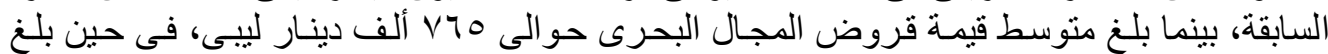

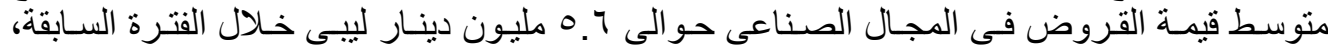

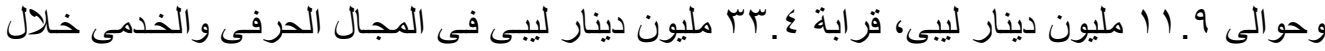
الفترة السابقة على الترتيب.

وفي ضوء ما أوضحه البحث من نتائج فإنه يوصي بالآتي:-

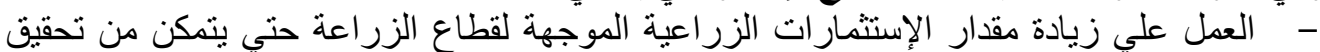

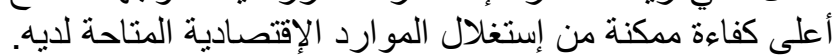

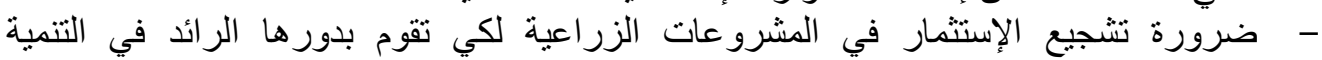

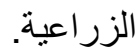

Fayoum J. Agric. Res. \& Dev., Vol. 27, No.1, January, 2013 
- - ضرورة توفير القروض الزراعية بشروط ميسرة لما لها من دور فعال في التنمية الزر اعية.

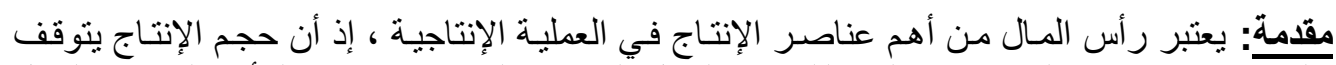

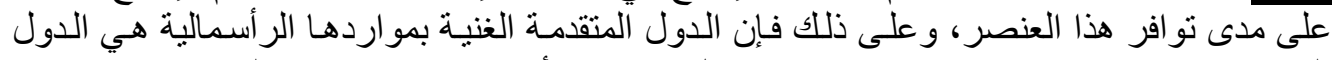

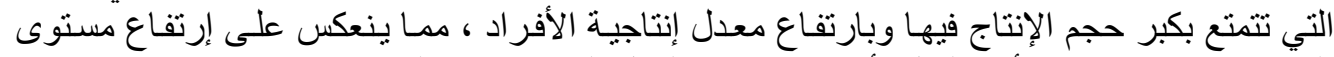

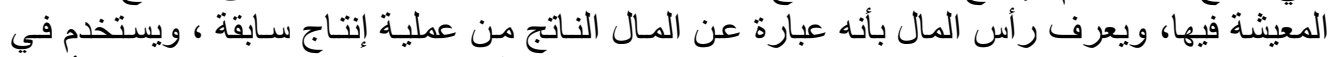

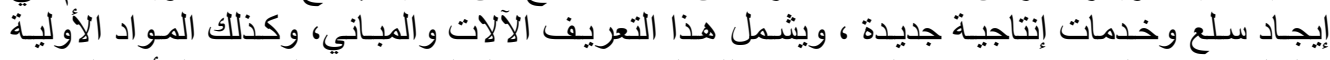

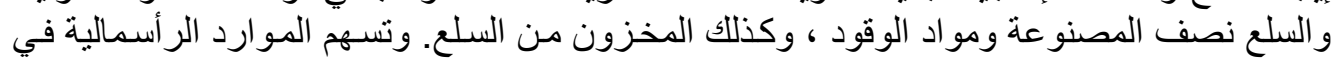

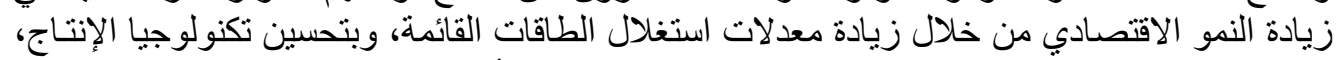

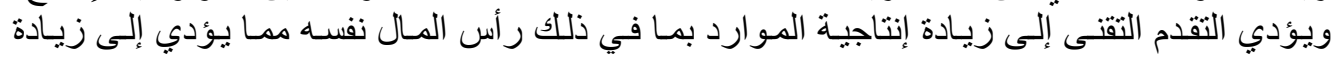

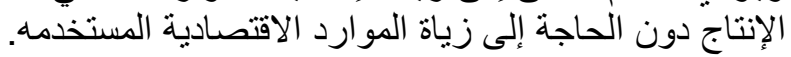

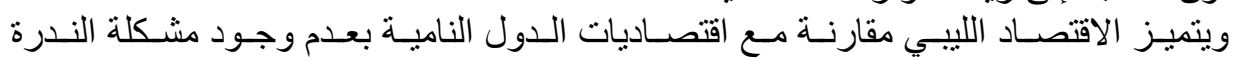

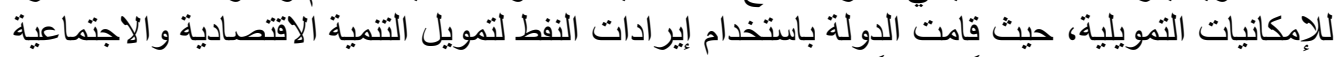

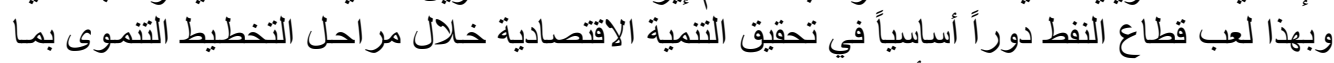

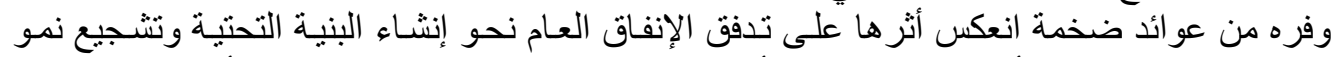

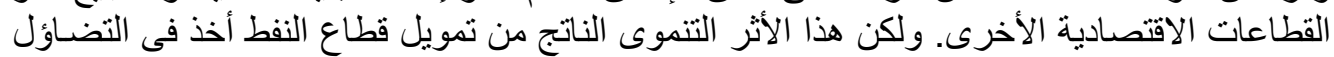

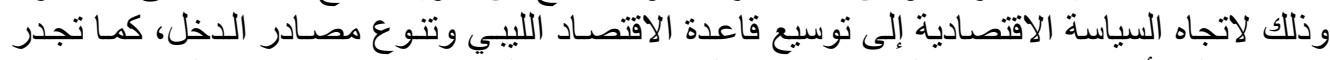

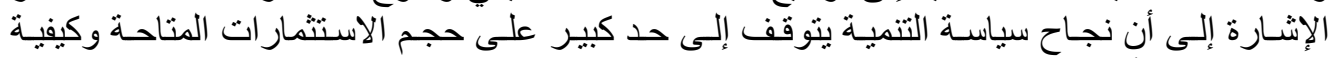

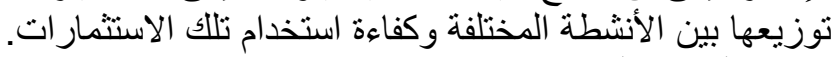

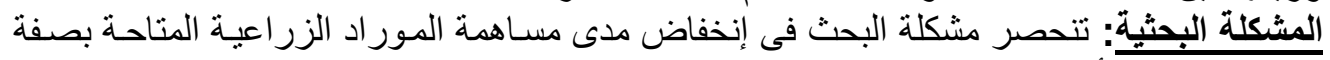

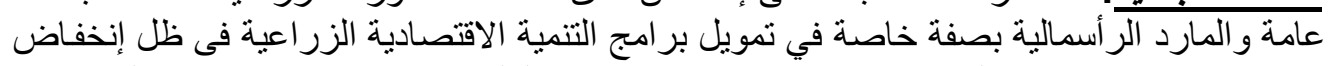

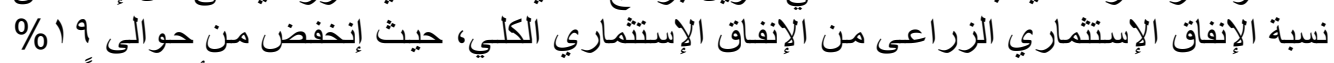

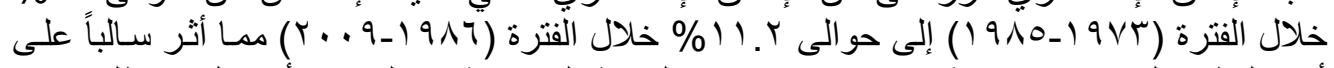

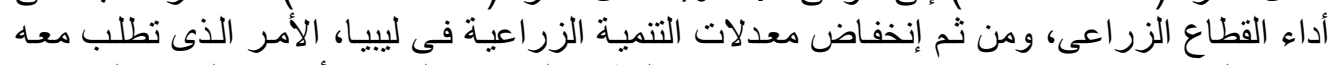

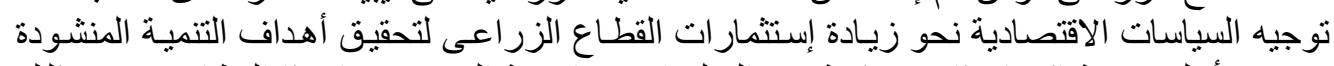

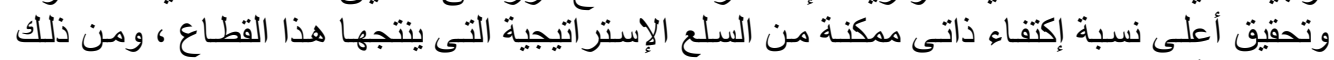

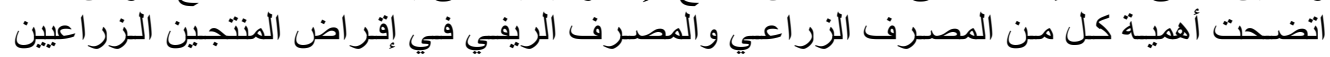

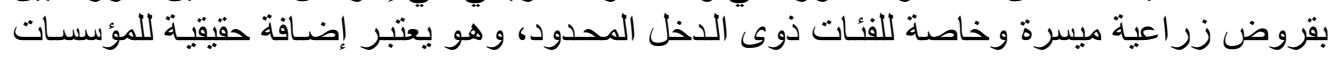

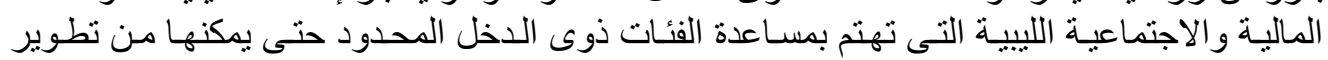

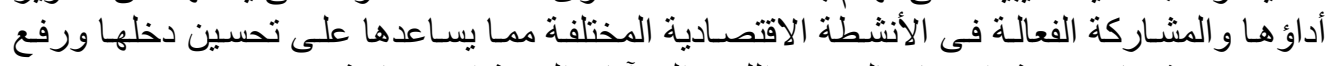

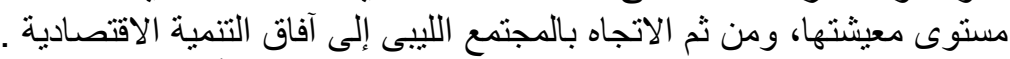

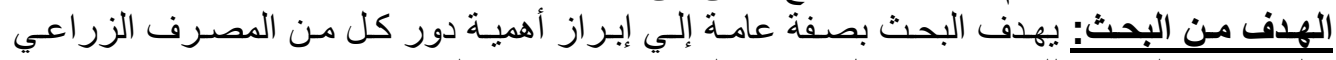

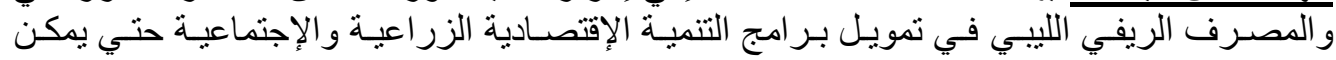

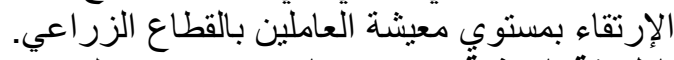

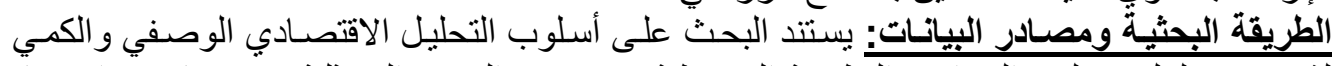

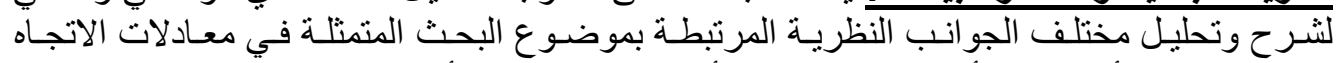

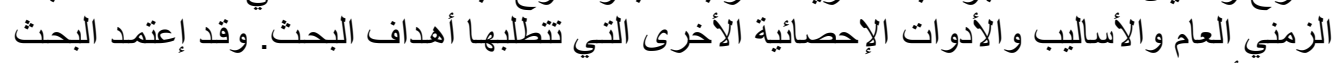

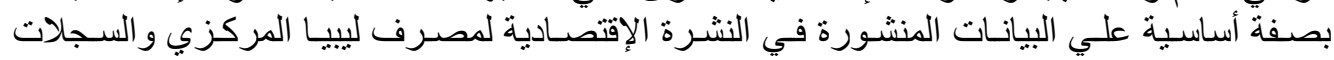

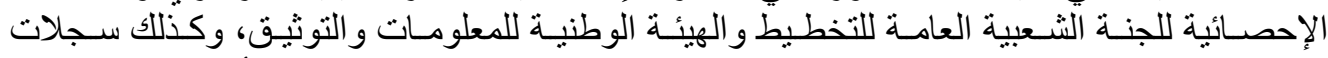

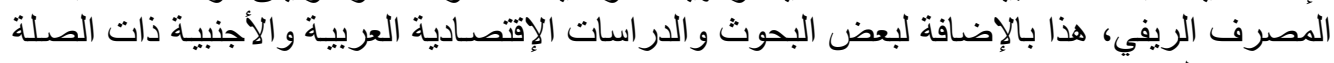
بموضوع البحث.

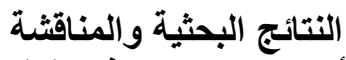

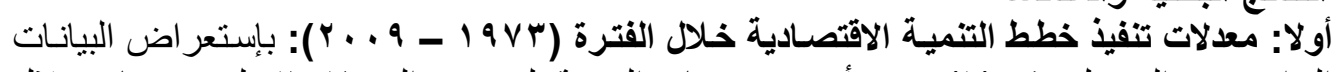

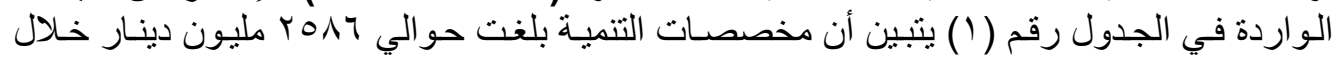

Fayoum J. Agric. Res. \& Dev., Vol. 27, No.1, January, 2013 


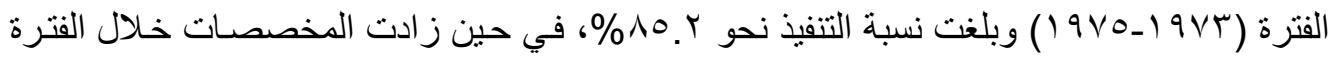

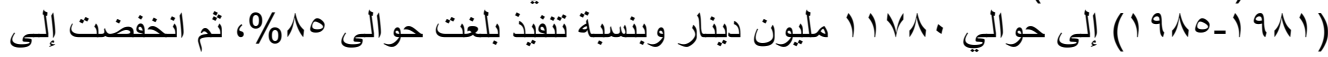

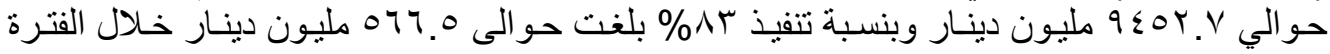

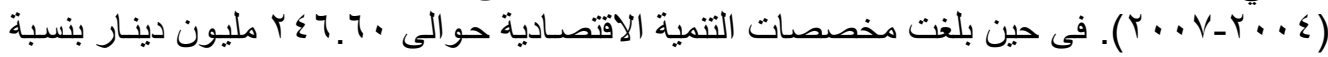

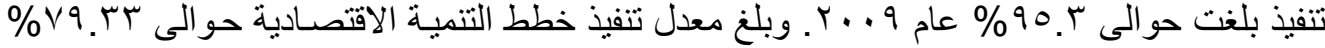

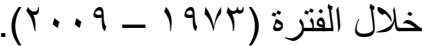

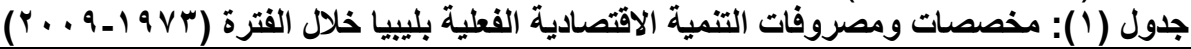

\begin{tabular}{|c|c|c|c|}
\hline معدل التنفيذ (\%) & المصروفات & المخصصات & البيان \\
\hline $10 . Y$ & Yr.T. & ro10.9 & $19 \vee 0_{-} 19 \mathrm{Vr}$ \\
\hline QY.V & Nroq. & $M \Lambda I T . Y$ & $191 .-19 \vee 7$ \\
\hline $9 \cdot .1$ & $1.79 r . \wedge$ & $11 \vee \wedge .$. & $1910_{-} 1911$ \\
\hline $0 \Lambda \mathrm{V}$ & $\varepsilon 10 \%$ & $V \cdot V 0.7$ & $199 .-1917$ \\
\hline$\sum 0 . V$ & rrol." & or $\$ 9.0$ & $1990-1991$ \\
\hline$V \Lambda . r$ & $\varepsilon r Y \Lambda . r$ & oorv. & r...-1997 \\
\hline$\wedge$. & VYIO.V & А9Y... & $r \ldots r-Y \ldots l$ \\
\hline$\lambda \mu$ & VQY... & qडOY.V & $r \ldots V_{-} r_{\ldots}$ \\
\hline 91 & TrY.9A & YTV.VT & $r \cdots \wedge$ \\
\hline 90.4 & YTO.IT & $r \leq 7.7$. & $r \ldots q$ \\
\hline *Vq rr & $\varepsilon \vee 0 q, 1 Y$ & $099 \wedge \vee 7$ & $r \ldots q-19 V r$ \\
\hline
\end{tabular}

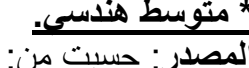

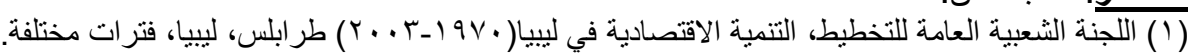

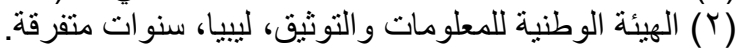

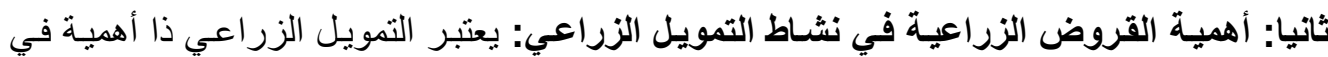

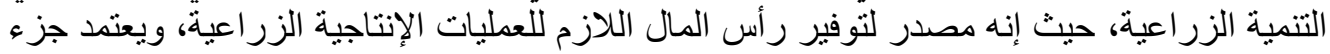

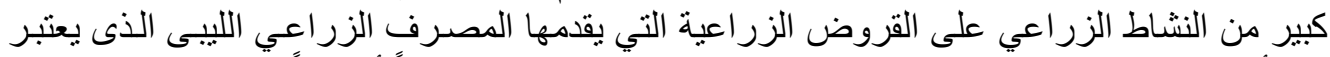

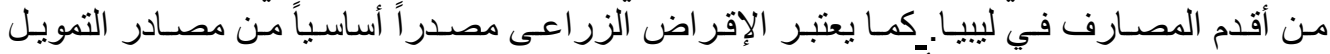

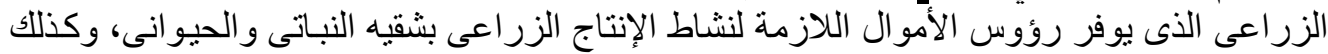

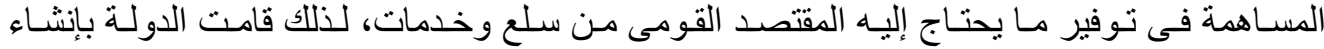

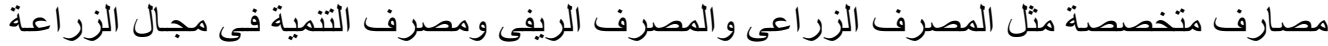

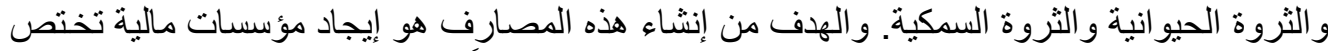

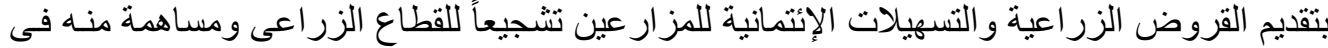

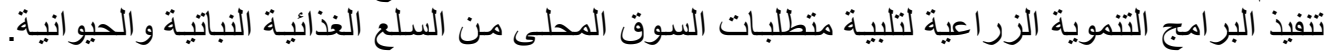

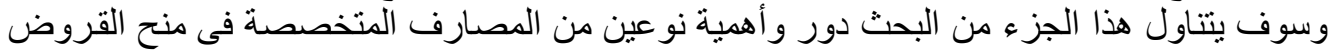

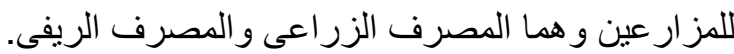

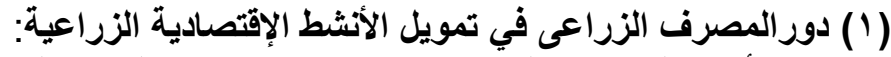

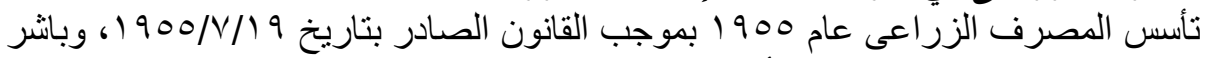

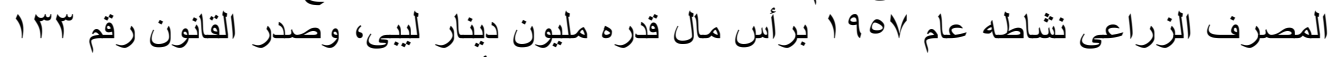

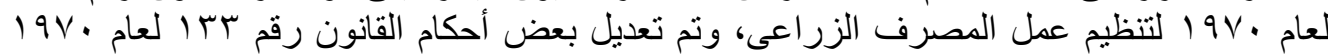

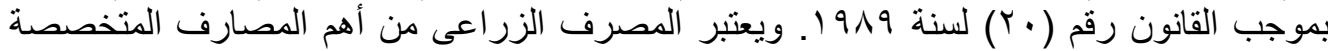

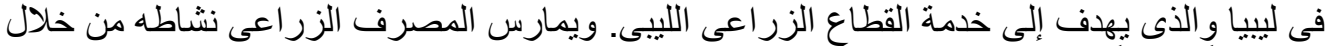

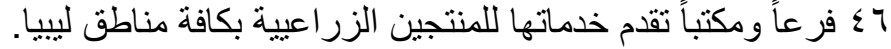

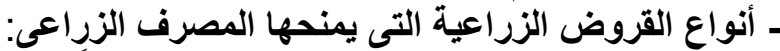

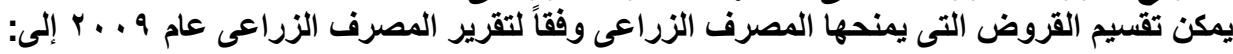

Fayoum J. Agric. Res. \& Dev., Vol. 27, No.1, January, 2013 
(أ) من حيث الآجال: يقدم المصرف الزر اعى ثلاثة أنواع رئيسية من القروض الزر اعية وفقاً لآجالها وهى:

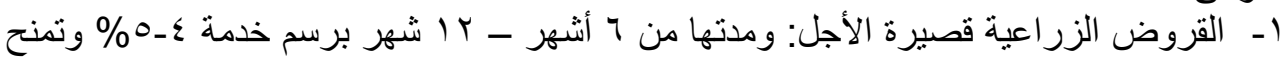

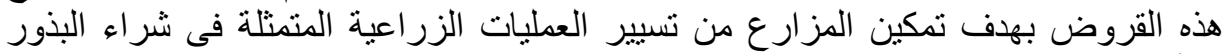

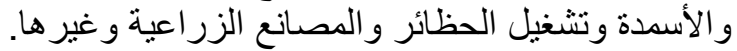

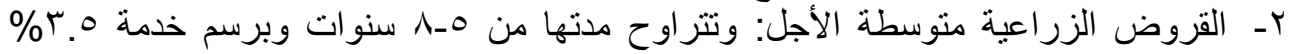

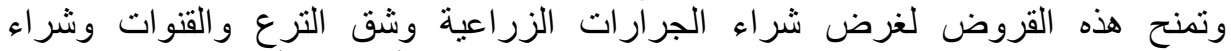

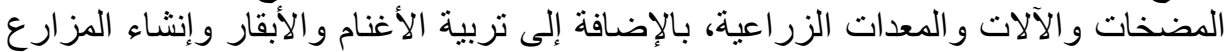

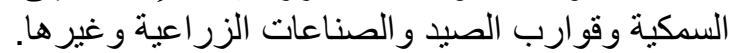

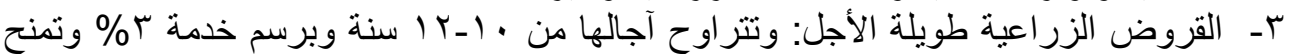

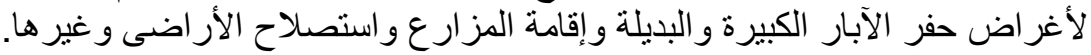

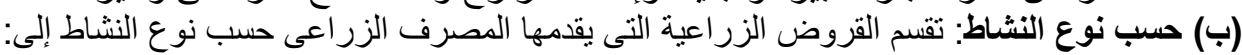

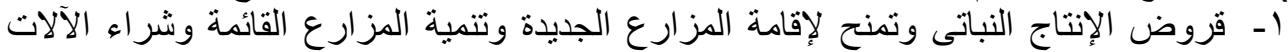

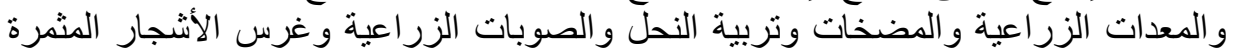

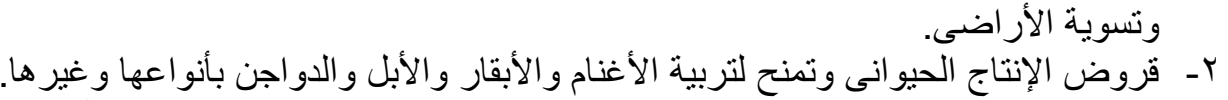

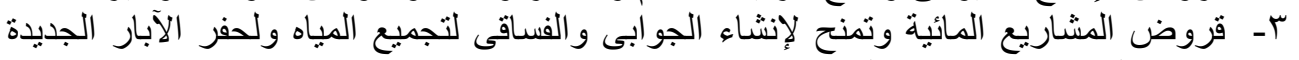

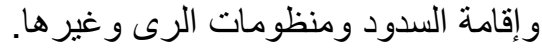

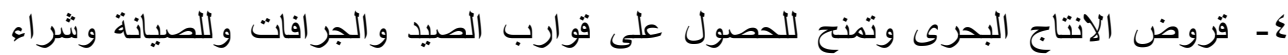

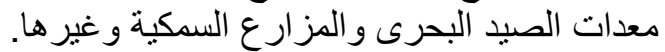

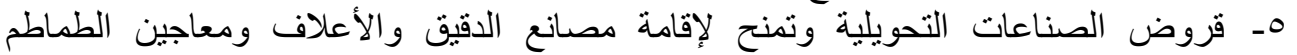

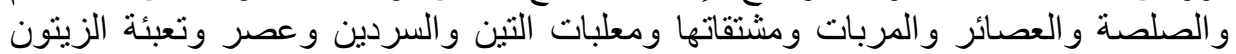
و وغير ها. 7- قروض التشغيل وتمنح لتشغيل المصانع وحظائر الدواجن وشراء الأعلاف و المواشىى

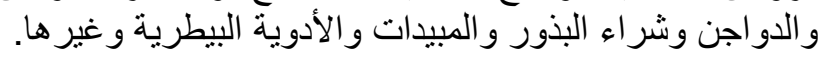

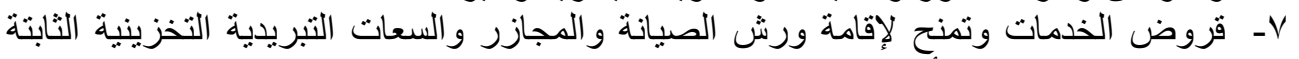

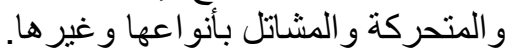

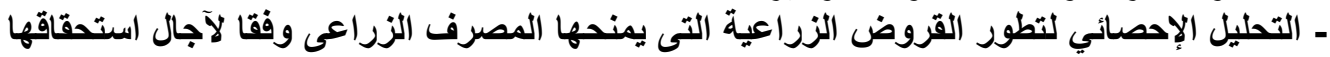

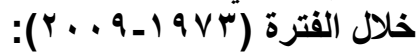

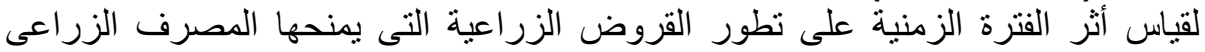

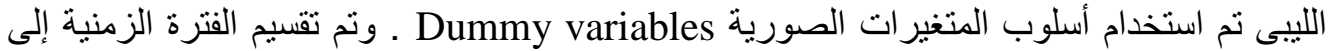

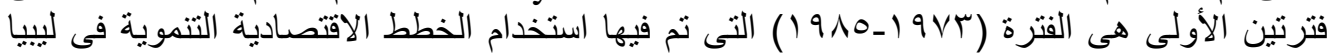

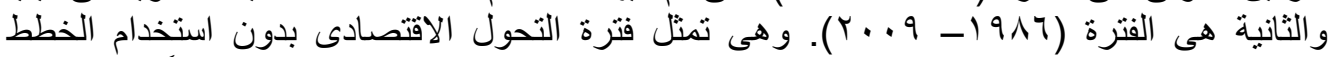

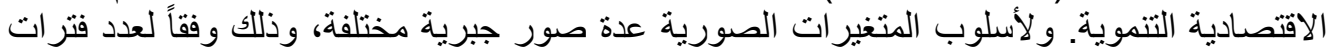

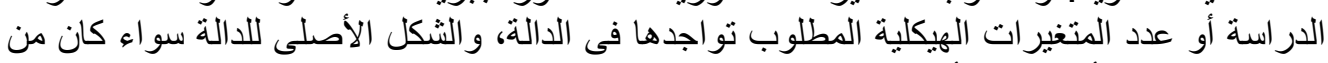

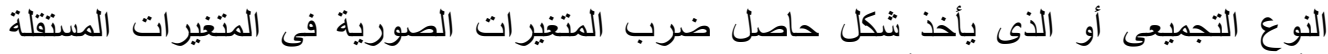

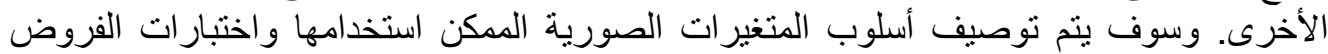

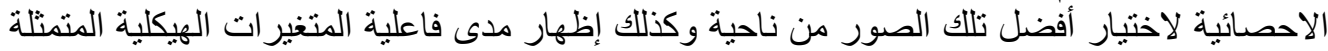

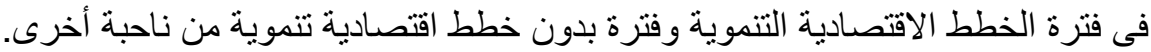

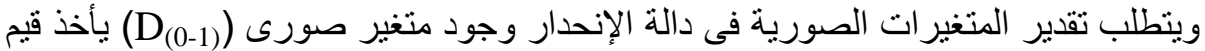

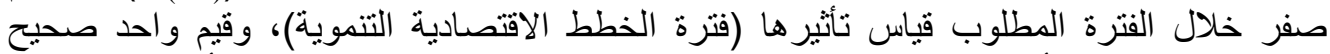

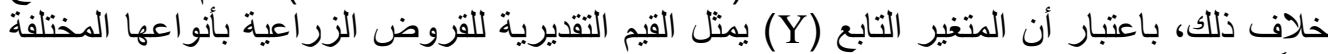

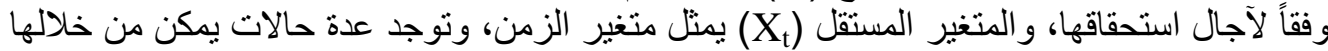

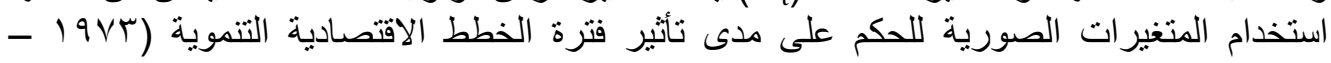

Fayoum J. Agric. Res. \& Dev., Vol. 27, No.1, January, 2013 
90

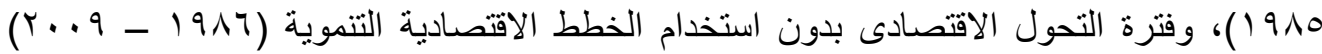

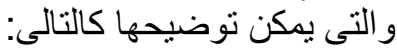

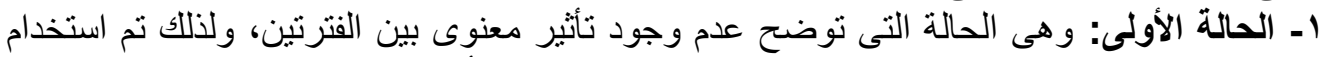

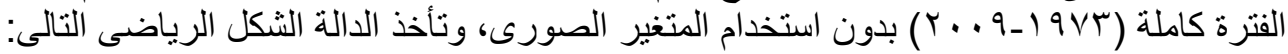
$\hat{\mathrm{Y}} \mathrm{t}=\mathrm{a}_{0}+\mathrm{Bo} \mathrm{X}_{\mathrm{t}} \rightarrow(1)$

Yـ الحالة الثانية: وهى تفترض وجود تأثبر معنوى بين الفترتين وتفترض تغير الحد الثابت للمعادلة

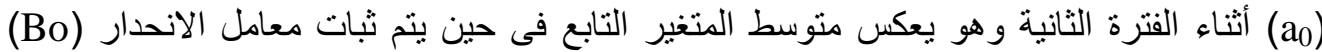

$\hat{\mathrm{Y}} \mathrm{t}=\mathrm{a}_{0}+\mathrm{a}_{1 \mathrm{D}}+\mathrm{Bo} \mathrm{X}_{\mathrm{t}} \rightarrow(2)$ وتأخذ الدالة الثكل الرياضى الفز التالى:

وللتأكد من تأثير فترة الخطط الاقتصادية التنموية وفترة التحول الاقتصادى يتم اختبار فرض

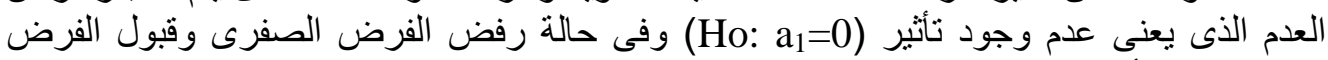
البديل بمعنى تأثثر تلك الفترنين على المتغير التابع ويتم تقسيم المعادلة (2) إلى معادلتين كالتالى

$\mathrm{Y}_{\mathrm{t}}=\mathrm{a}_{0}+\mathrm{Bo} \mathrm{X}_{\mathrm{t}}$ الفترة الأولى لفئ

Y

ـ أثر التحولات الاقتصادية على القروض الزراعية المقدمة من المصرف الزراعى:

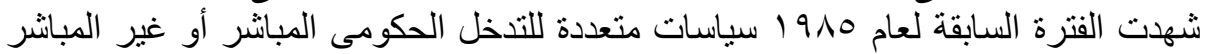

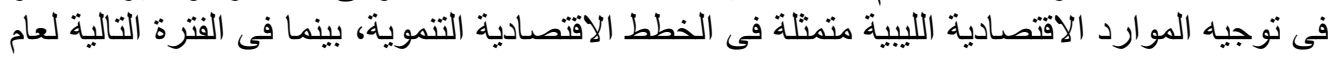

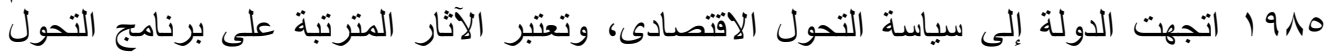

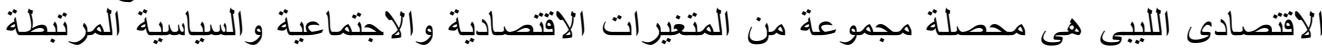
بتلك التحو لات

وللتعرف على أثز التحولات الاقتصادية الايجابية و السلبية على القروض الزر اعية المقدمة

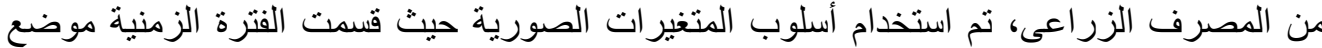

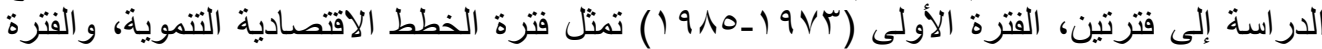

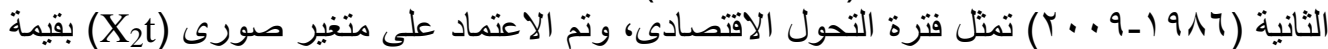

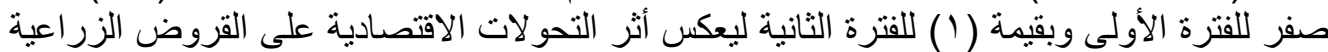

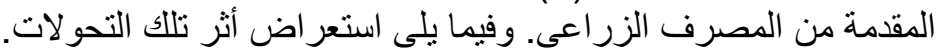

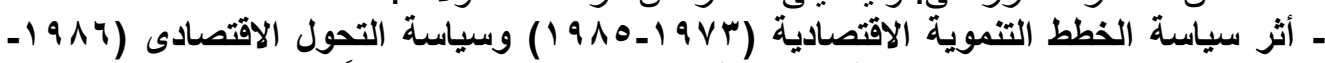

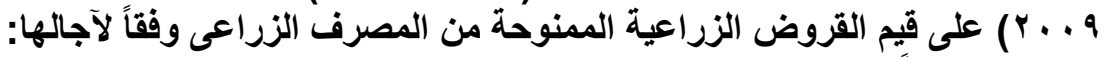

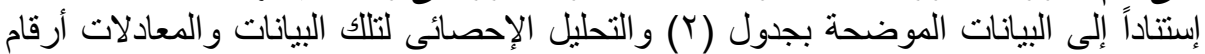

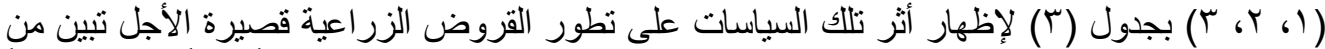

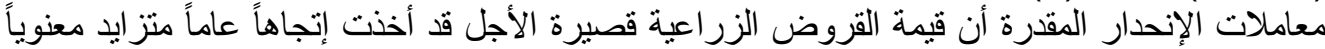

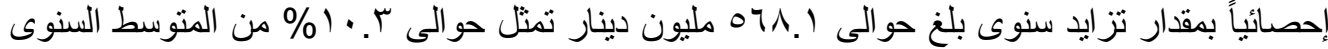

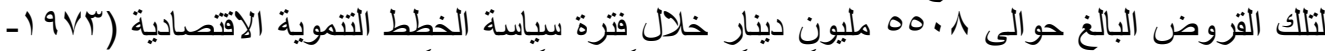

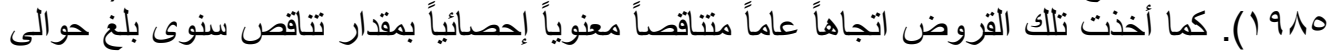

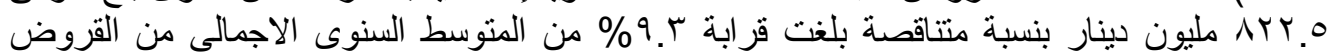

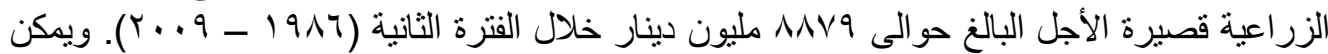

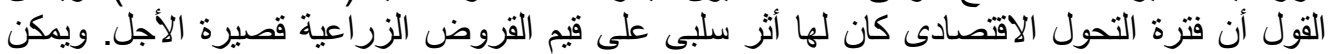

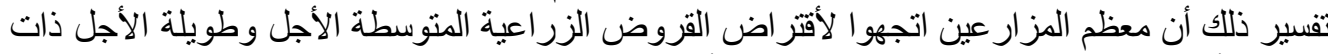

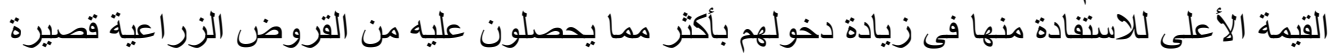
الأجل.

Fayoum J. Agric. Res. \& Dev., Vol. 27, No.1, January, 2013 
94

ولمعرفة أثر تلك السياسات السابق ذكرها على تطور القروض الزرواعبة منوسطة الأجل

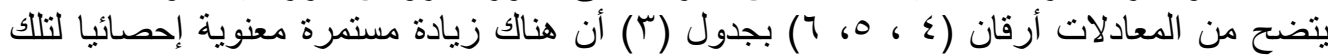

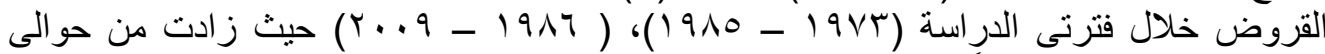

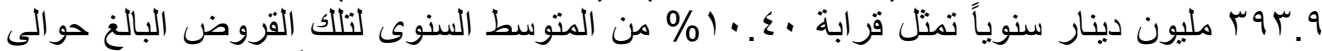

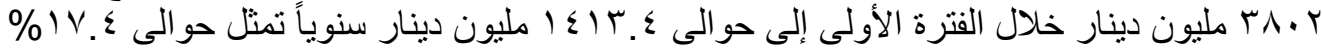

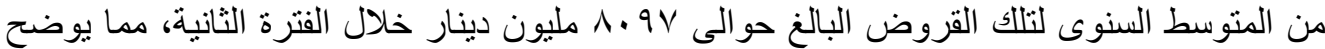

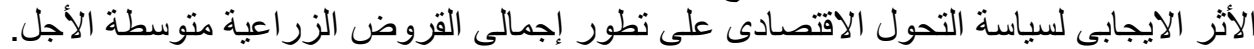

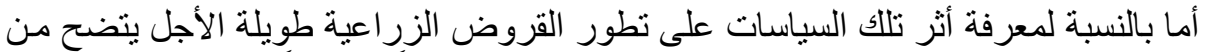

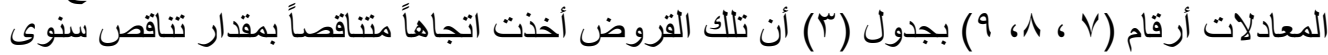

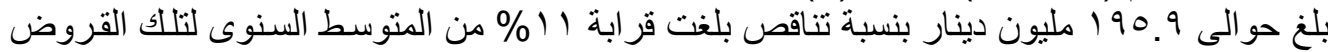

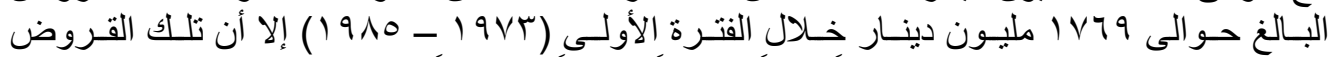

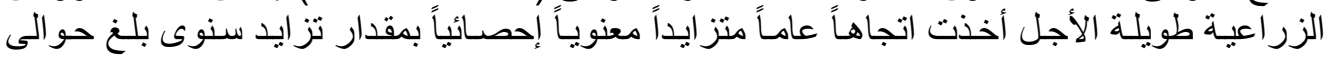

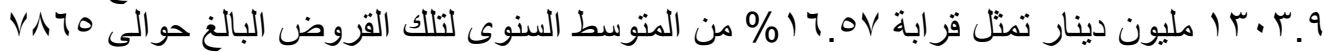

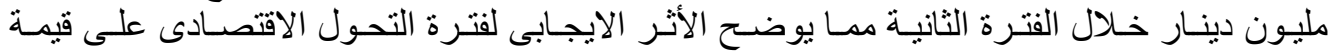

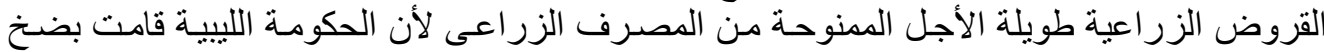

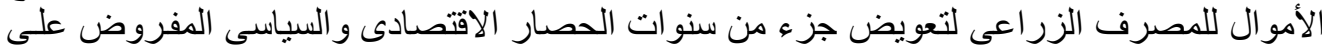

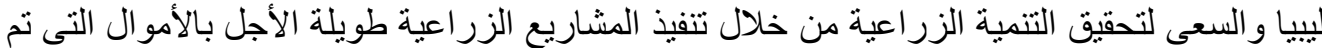

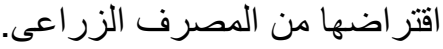

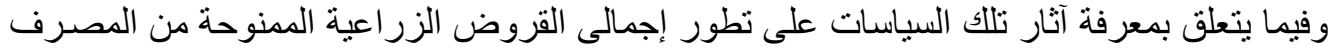

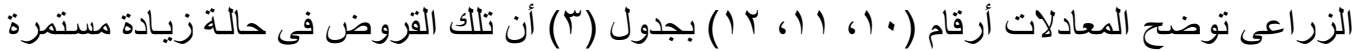

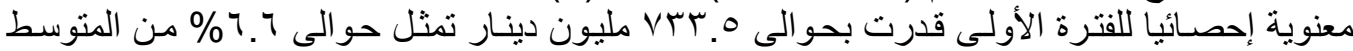

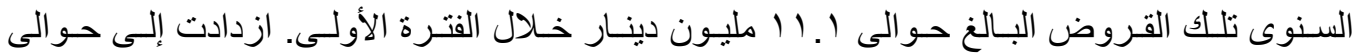

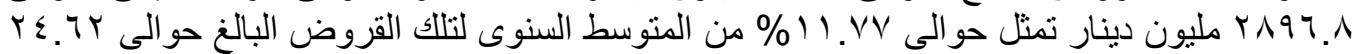

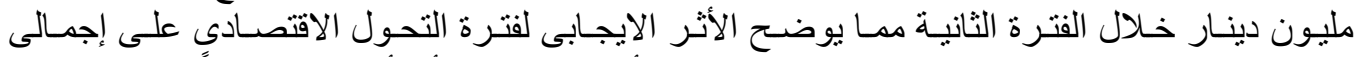

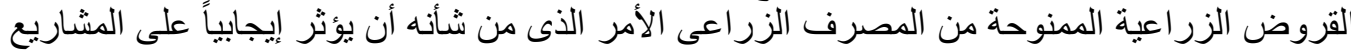

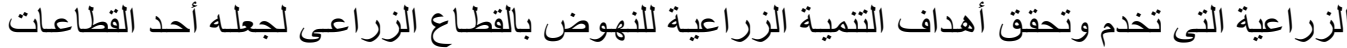

(Y) دورالمصرف الريفي في تمويل الأنثط الإقتصادية الزراعية:

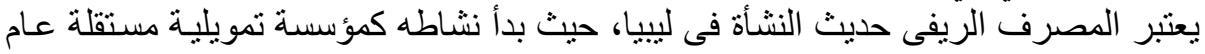

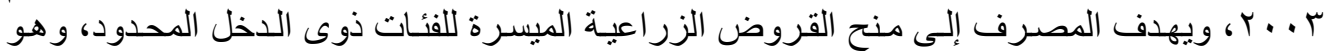

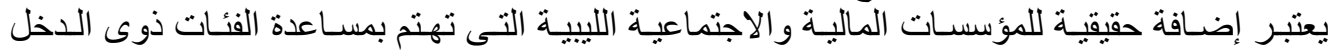

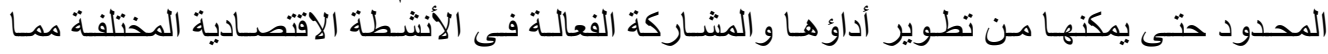

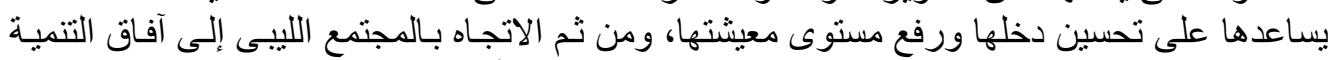

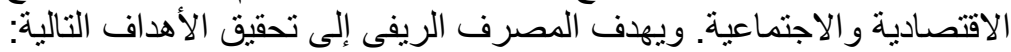

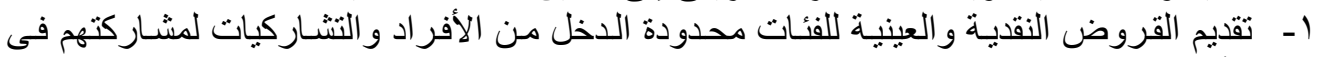

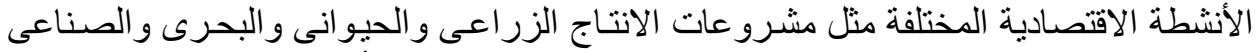

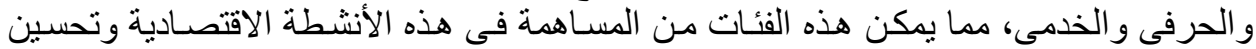

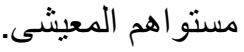

r- جذب الاستثمار ات الأجنبية بهدف تمويل المشرو عات الانتاجية فى قرى وريف ليبيا.

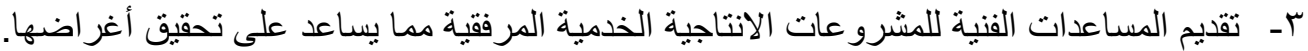

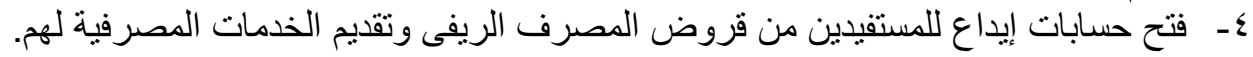

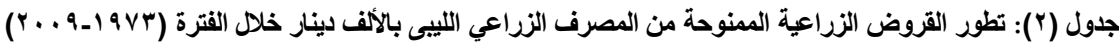

\begin{tabular}{|c|c|c|c|c|}
\hline إجمالى القروض الزراعية & طويلة الأجل & متوسطة الأجل & قصيرة الأجل & السنوات \\
\hline$V \leqslant r 4$ & $1 \leq \leqslant 0$ & $r r \cdot \Lambda$ & rVAr & $19 V \%$ \\
\hline
\end{tabular}

Fayoum J. Agric. Res. \& Dev., Vol. 27, No.1, January, 2013 
$9 \vee$

\begin{tabular}{|c|c|c|c|c|}
\hline 11199 & 1940 & $\Sigma V \cdot \Sigma$ & ¿OV. & $19 V \leqslant$ \\
\hline A9YT & $11 K V$ & rq10 & r人I & 1980 \\
\hline ^ᄉ०. & Tा & 1940 & rNIT & 1987 \\
\hline $1 \ldots 1 \varepsilon$ & Y7.0 & $1 \wedge r q$ & $00 \mathrm{~V}$. & $198 V$ \\
\hline $1 \leq \Lambda I V$ & IVT & 7811 & TrEY & $19 \vee \wedge$ \\
\hline 11101 & 1.9. & $\varepsilon \cdot M$ & $\Lambda \cdot r V$ & 1979 \\
\hline $011 Y$ & NTE & loVT & $r V \cdot T$ & $19 \wedge$. \\
\hline lorVE & INH人 & Y97. & $1.0 V 7$ & 1911 \\
\hline $1 \leqslant 004$ & rrvq & $\varepsilon 0 \vee \leqslant$ & $77 \ldots$ & $19 \wedge \mathrm{r}$ \\
\hline $1194 \varepsilon$ & $1 \wedge Y \varepsilon$ & $7 \varepsilon \ldots$ & $r v \ldots$ & $19 \wedge r$ \\
\hline Irogr & $1 \cdot \sum Y$ & rqA. & VOV. & $19 \wedge \varepsilon$ \\
\hline $1 \cdot r \cdot r$ & דוצו & Tos. & OOYV & 1910 \\
\hline $111 \ldots$ & 1.879 & r.A.. & $0.0 . V$ & $\left(19 \wedge 0_{-} \mid 9 \vee T\right)$ \\
\hline 9ห71 & $1 \wedge \wedge$. & $\varepsilon \leqslant Y \wedge$ & r90r & $19 \wedge 7$ \\
\hline $11 \leqslant \mu \wedge$ & TVOT & $\varepsilon \cdot Y_{0}$ & ะ77. & $19 \wedge V$ \\
\hline $17 \ldots$ & $\leqslant \wedge 0$. & Tor. & 纟าT. & $19 \wedge 1$ \\
\hline आイו & 99Tr & AVVO & ITIKE & 1919 \\
\hline $19 . \leqslant 0$ & $7 \wedge \cdot \varepsilon$ & $\varepsilon V V$. & $V \leqslant V I$ & 199. \\
\hline $1 \wedge 0 \leqslant V$ & ro. & 1090 & $11 \leq \varepsilon \leq$ & 1991 \\
\hline ITHA & YA.O & rrv. & 7.74 & 1994 \\
\hline 17.01 & $97 V$ & $r .9 \leq$ & IY99V & 1994 \\
\hline 11941 & VTO & 1790 & 9491 & 1995 \\
\hline $1110 r$ & $r .9 r$ & Yフ7. & ITE.. & 1990 \\
\hline YE.VY & КАT & $\wedge \vee 1$. & $110 \ldots$ & 1997 \\
\hline IAVAY & YITE & $\pi Y \cdot 1$ & $1 \cdot \varepsilon \varepsilon$. & 1998 \\
\hline r. $9 \varepsilon$. & M... & $01 \pi$. & IYVI. & 1991 \\
\hline YVY.. & $r \ldots$ & $1.0 \ldots$ & $1 \leqslant V \ldots$ & 1999 \\
\hline rVะ.. & $1 \leq \ldots$ & I. T. & rOA.. & $r \ldots$ \\
\hline r7o.. & Tr.. & $111 \ldots$ & $194 \ldots$ & $r \ldots 1$ \\
\hline $1110 \ldots$ & $00 \ldots$ & $0 \leqslant 0 \ldots$ & $9 \ldots$ & $r \ldots r$ \\
\hline$\vee 9991$ & OYYYI & $T \varepsilon \cdot T V$ & MVI. & $r \ldots r$ \\
\hline V^०9 & קות. & YVV. & $1 V Y 9$ & $r \ldots \varepsilon$ \\
\hline Irov. & 1179 & $r \leqslant \wedge 0$ & 9917 & $r \ldots o$ \\
\hline 1Tro. & $\wedge \leq 9$. & ґАт. & $1 \ldots$ & $r \ldots T$ \\
\hline ITros & $1 . r \leq$ & Аรт. & Yフ7. & $r \ldots v$ \\
\hline $1 . r \wedge V$ & ז... & ro.. & TrAV & $r \ldots \lambda$ \\
\hline$\varepsilon \wedge \mid \wedge$ & $r q \ldots$ & $7 \ldots$ & $1 \pi 11$ & $r \ldots q$ \\
\hline$r \leq 719$ & จ^ท० & $\Lambda .9 V$ & $\wedge \wedge \vee q$ & $(Y \ldots 9-19 \wedge 7)$ \\
\hline $19 \wedge 41$ & $007 V$ & $70 \wedge \wedge$ & 1790 & $(r \ldots 9-19 \vee r)$ \\
\hline
\end{tabular}

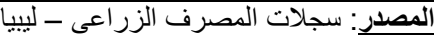

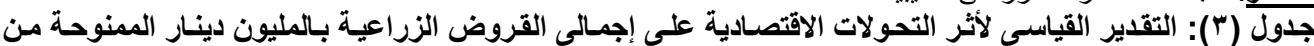

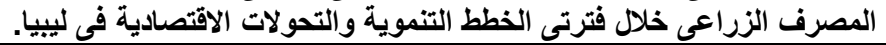

\begin{tabular}{|c|c|c|c|c|}
\hline النموذج & $\mathbf{F}$ & $\mathbf{R}^{2}$ & النموذج & المتغير التابع \\
\hline $\begin{array}{l}1 \\
\dot{r} \\
r\end{array}$ & 110.7 & .91 & $\begin{aligned} \hat{\mathrm{Y}} & =1531.2+568.1 \mathrm{~T}+28321 \mathrm{D}_{1}-1390.6 \mathrm{D}_{2} \\
(17.69) \quad(4.67) & (-10.63) \\
\hat{\mathrm{Y}} & =1531.2+568.1 \mathrm{~T} \\
\hat{\mathrm{Y}} & =4063.3-822.5 \mathrm{~T}\end{aligned}$ & الفترة الأولى قصيرة الأجل \\
\hline $\begin{array}{l}\varepsilon \\
0 \\
7\end{array}$ & $1 \leqslant . r$ & $\because \leqslant 0$ & $\begin{array}{l}\hat{\mathrm{Y}}=3802.2+393.9 \mathrm{~T}-21726.5 \mathrm{D}_{1}+1020.4 \mathrm{D}_{2} \\
\quad(0.76) \quad(-3.81) \\
\hat{\mathrm{Y}}=3802.2+393.9 \mathrm{~T} \\
\hat{\mathrm{Y}}=1627.7+1414.3 \mathrm{~T}\end{array}$ & الفترة الأولى متوسطة الأجل \\
\hline
\end{tabular}

Fayoum J. Agric. Res. \& Dev., Vol. 27, No.1, January, 2013 
91

\begin{tabular}{|c|c|c|c|c|}
\hline $\begin{array}{l}V \\
\wedge \\
q\end{array}$ & 17.8 & $\because 0$ & $\begin{array}{l}\hat{\mathrm{Y}}=1786.3-195.9 \mathrm{~T}-30956.8 \mathrm{D}_{1}+1499.8 \mathrm{D}_{2} \\
\quad(-0.27) \quad(-3.93) \\
\hat{\mathrm{Y}}=1786.3-195.9 \mathrm{~T} \\
\hat{\mathrm{Y}}=-29170.5+1695.7 \mathrm{~T}\end{array}$ & الفترة الأولى طويلة الأجل \\
\hline $\begin{array}{l}1 . \\
11 \\
11\end{array}$ & 11 &.$r q$ & $\begin{aligned} \hat{\mathrm{Y}}=11096.5+ & 773.5 \mathrm{~T}-38500 \mathrm{D}_{1}+2096.3 \mathrm{D}_{2} \\
& (0.58) \quad(-2.62) \\
\hat{\mathrm{Y}}=11096.5+773.5 \mathrm{~T} & (4.0) \\
\mathrm{Y} & =-27403.5+2869.87 \mathrm{~T}\end{aligned}$ & 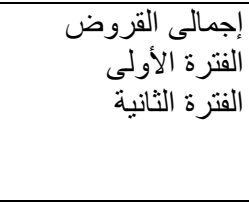 \\
\hline
\end{tabular}

حيث

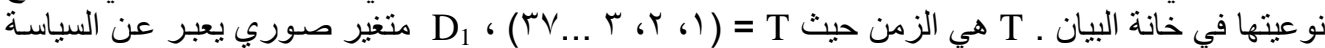

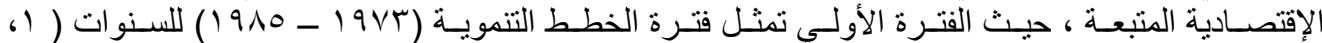

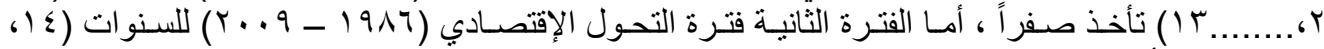

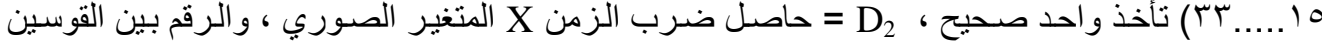

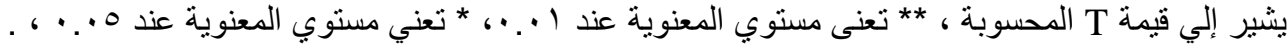

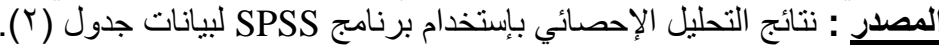

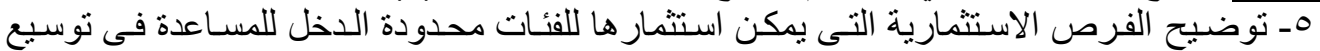

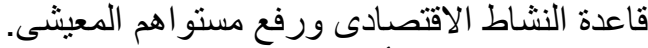

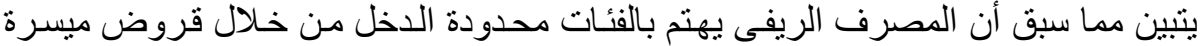

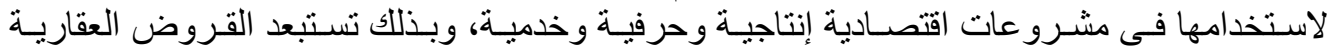

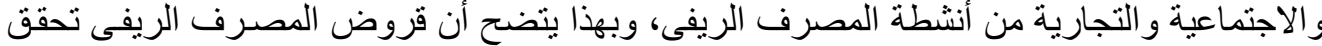

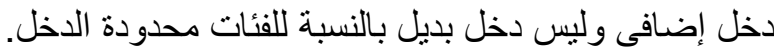
ـ أنواع قروض المصرف الريفى:

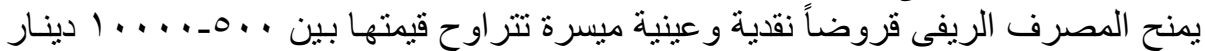

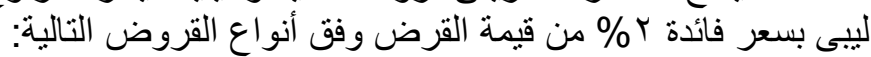

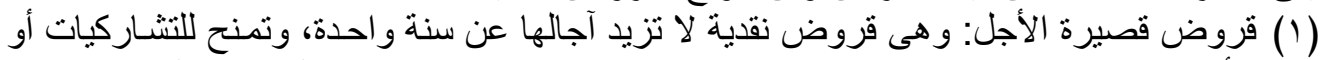

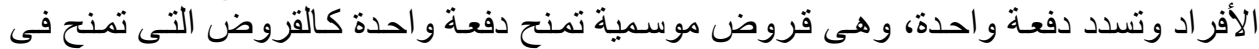

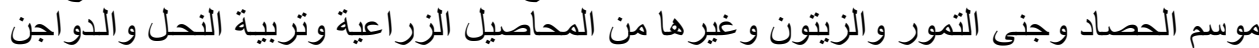

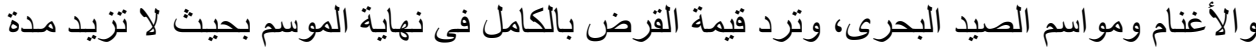
القروض الموسمية عن سنة و احدة.

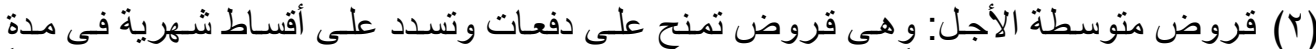

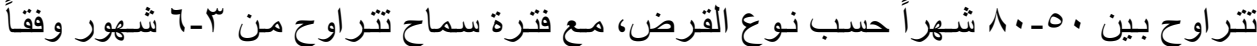

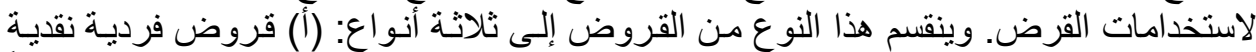

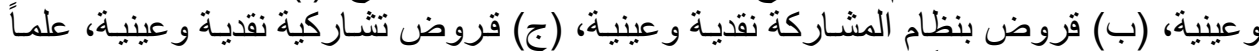

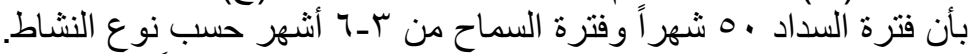

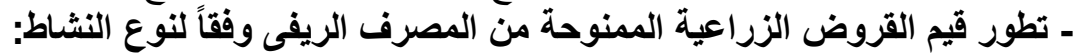

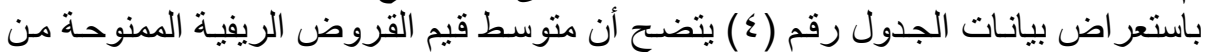

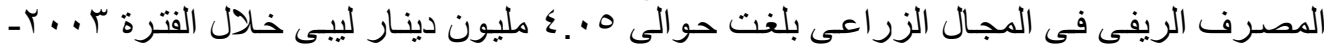

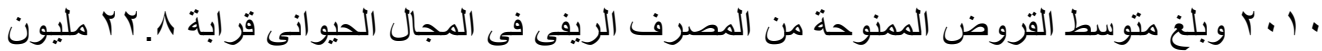

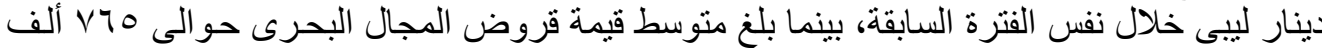

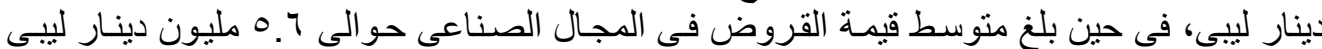

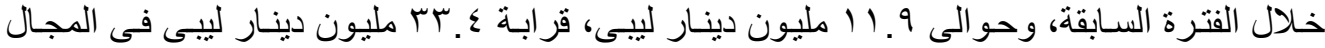
الحرفى و الخدمى خلال الفترة السابقة على الترتيب.

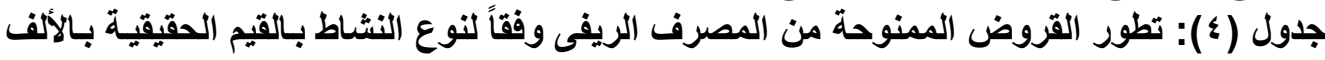

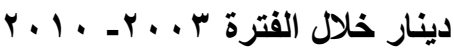

Fayoum J. Agric. Res. \& Dev., Vol. 27, No.1, January, 2013 
99

\begin{tabular}{|c|c|c|c|c|c|c|c|c|c|c|c|c|c|}
\hline المجموع & $\%$ & خلمى & $\%$ & حرفى & $\%$ & صناعى & $\%$ & بحرى & $\%$ & زراعى & $\%$ & حيوانى & السنـه \\
\hline $111 \leqslant r$ & $r$ & r91 & $\varepsilon$ & $\varepsilon .9$ & $r$ & T17 & 1 & $\varepsilon$. & 11 & IYVT & $V \lambda$ & AV.r & T..T \\
\hline$r \leqslant r \leqslant q$ & 11 & rVAT & 1. & rTVA & $r$ & 1104 & $r$ & $\sum \lambda 1$ & 9 & $r 1 \leqslant 9$ & 70 & TY\&.r & T.. \\
\hline TKVT. & $\pi$ & 11109 & 1. & $1 \pi 1 V$ & $\varepsilon$ & OTYA & $T$ & $r \leqslant Y$ & 0 & 0971 & IV & YIVO9 & r... \\
\hline 170011 & $r$. & $011 \mathrm{~T}$ & IV & YNTVT & $\mathrm{V}$ & $11 \leqslant r 9$ & $r$ & rrol & $\varepsilon$ & $V \leqslant \varepsilon$. & rq & $7 r \lambda .9$ & Y..T \\
\hline $1 \leq .110$ & 0. & $V .7 / 7$ & 10 & $r \cdot r \leqslant 1$ & $\Lambda$ & $117 \leqslant r$ & 1 & $9 \leqslant 1$ & $\varepsilon$ & $7.7 V$ & Tr & $r .0 . V$ & $r \ldots V$ \\
\hline ror II & $\varepsilon \wedge$ & $17 \varepsilon \cdot V$ & 17 & $0 r \leqslant \varepsilon$ & 7 & YIOV & 1 & $1 \leqslant$. & 0 & 187. & $r \varepsilon$ & $\Lambda \varepsilon \cdot r$ & $r \ldots \lambda$ \\
\hline $7 r .19$ & $\leqslant 7$ & rqYqr & YI & $1 \pi \mid \leq \varepsilon$ & 1. & $T r \leqslant 0$ & 1 & 711 & $\varepsilon$ & FTYA & 11 & 11.71 & $r \ldots q$ \\
\hline $0 Y 117$ & TV & $1 \leqslant Y T_{0}$ & Y) & 11.05 & $1 \pi$ & 779. & 1 & $r .9$ & $\Lambda$ & \&10r & $r$. & $107 \mathrm{~V}$. & r.1. \\
\hline VAOYA.VO & $\varepsilon r$ & TrTAo & 10 & $119 . Y .1 T$ & v & OTrY.0 & 1 & V7O.YO & 0 & $\varepsilon .0 \leqslant . Y_{0}$ & rq & TYV9O & السنوي ســ \\
\hline
\end{tabular}

المصرف الريفى - التقرير السنوى ـ أعداد متفرقة.

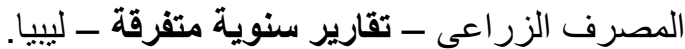

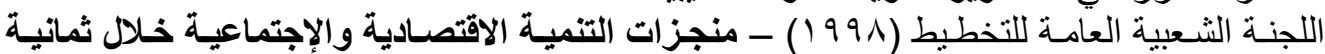

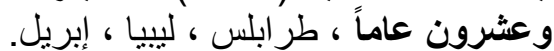

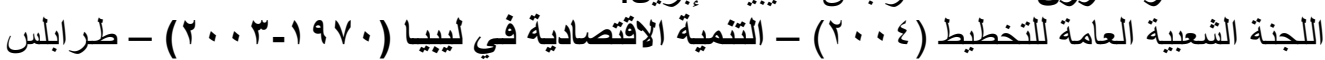

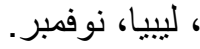

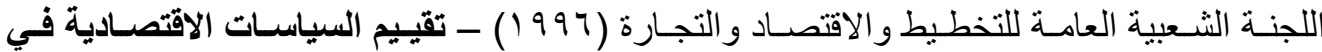

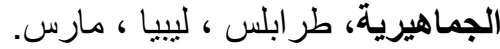

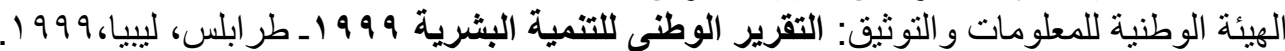

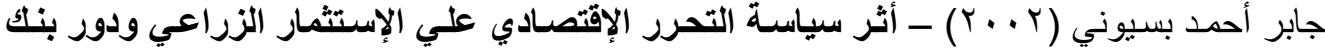

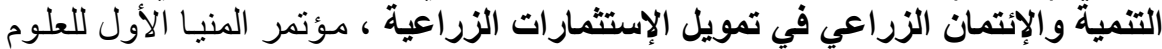

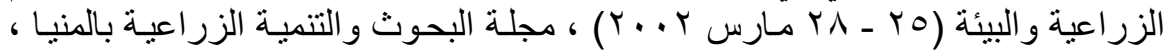

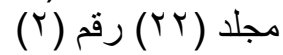

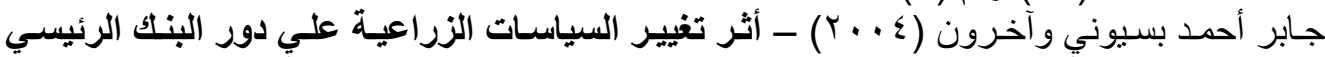

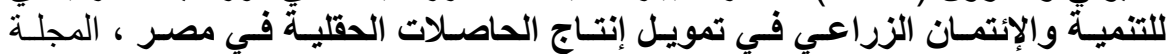

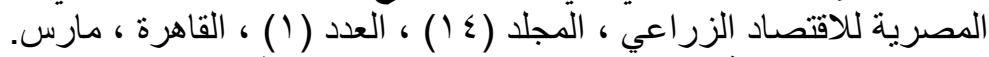

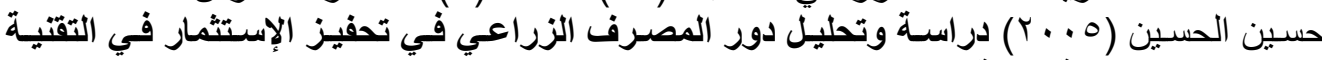

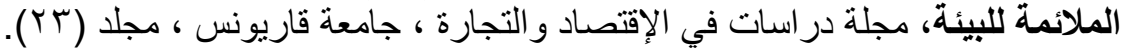

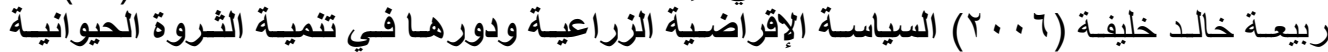

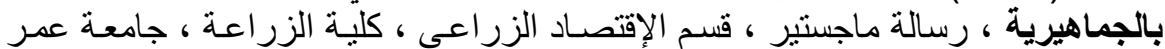

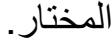

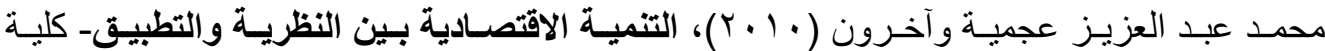
التجارة-جامعة الاسكندرية.

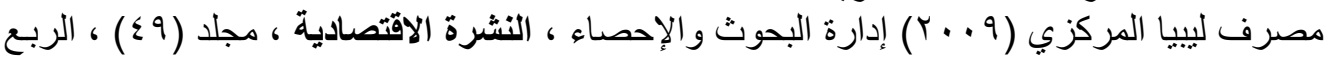

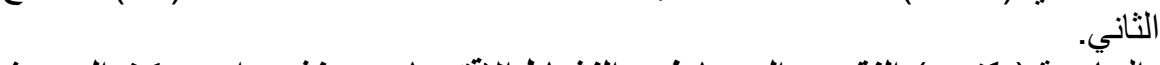

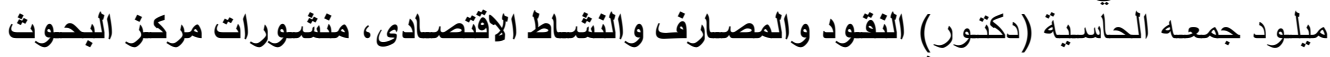

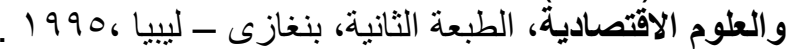

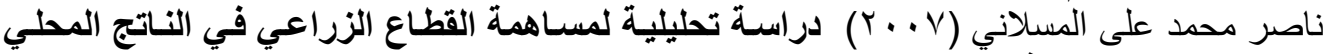

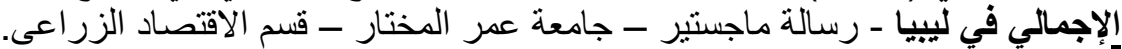

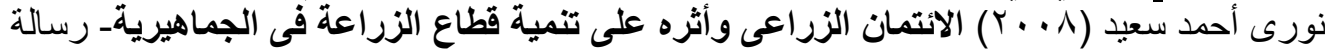

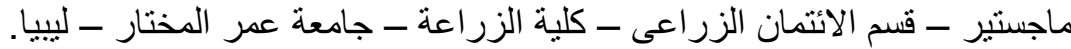

Barry R. and Ralph M., (1988) Quantitative Analysis for Management, $3^{\text {rd }}$ Edition, Allyn Baron Inc., U.S.A.

Fayoum J. Agric. Res. \& Dev., Vol. 27, No.1, January, 2013 
$1 \ldots$

Brillinger, David R., (1963) Time Series Data Analysis and Theory, Holt, Rinehart and Winstor, N.Y.

Draper, N.R. and H. Smith, (1977) Applied Regression Analysis, Wiley, New York.

Johnston, J., (1963) Econometric Methods, McGraw-Hill Book Co., New York.

\section{THE IMPORTANCE OF AGRICULTURAL AND THE RURAL BANK IN FINANCING AGRICULTURAL DEVELOPMENT EXPENDITURE IN LIBYA} ABSTRACT

The research problem is confined to decline in the contribution of agricultural resources generally available, and capitalist resources particularly to finance of agricultural economic development programs under the low proportion of agricultural investment expenditure of the total investment expenditure, which decreased from about 19\% during the period (1973-1985) to about $11.2 \%$ during the period (1986-2009), which impact negatively on the performance of the agricultural sector, and then low rates of agricultural development in Libya, which required him directing economic policies towards increasing investments of the agricultural sector to achieve the objectives of the desired development, achieve the highest rate of selfsufficiency possible strategic commodities produced by this sector, and it became clear the importance of each of the Agricultural Bank and Rural Bank in lending to agricultural producers loans agricultural accessible and special categories of low-income, which is a real addition to financial institutions and social Libyan interested in assisted groups low-income so that they can develop their performance and active participation in various economic

Fayoum J. Agric. Res. \& Dev., Vol. 27, No.1, January, 2013 
$1 \cdot 1$

activities, helping them to improve their income and raise their standard of living, and then trend Libyan community to the economic development prospects.

The research aims in general to highlight the importance of the role of each of the Agricultural Bank and the Libyan Rural Bank in financing agricultural economic development programs, social, even can raise the level of living of employees in the agricultural sector.

A study the evolution of the value of short-term agricultural loans it took years growing trend statistically significantly increased by increasing an annual rate of about 568.1 million dinars, representing about $10.3 \%$ of the annual average of such loans of about 5508 million dinars during the period of the policy of economic development plans (1973-1985). It also took those loans-year declining trend statistically significantly by decreasing the annual rate of about 822.5 million dinars by decreasing amounted to approximately $9.3 \%$ of the total annual average of short-term agricultural loans of about 8879 million dinars during the second period (1986 - 2009). It could be argued that the period of economic transformation has had a negative impact on the values of short-term agricultural loans. This can be explained that most of the farmers turned to borrow medium-term agricultural loans and long-term with the highest value to take advantage of them to increase their incomes more than what they get from the short-term agricultural loans.

As shown by the study of the evolution of the value of medium-term agricultural loans that there is a continuous increase significantly statistically for such loans during the two periods of the study (1973 - 1985), (1986 2009), increased from about 393.9 million dinars annually representing approximately $10.40 \%$ of the annual average for those loans of about 3802 million dinars during the first period to about 1.4134 billion dinars annually representing about $17.4 \%$ of the annual average of such loans of about 8097 million dinars during the second period, which demonstrates the positive impact of the policy of economic transformation on the evolution of the total medium-term agricultural loans.

As shown by the study of the evolution of the value of long term agricultural loans that those loans took a decreasing trend by decreasing annual rate of about 195.9 million dinars by decreasing amounted to approximately $11 \%$ of the annual average of such loans of about 1769 million dinars during the first period (1973 - 1985), but the those long-term agricultural loans took a general trend growing significantly statistically by growing an annual rate of about 1.3039 billion dinars, representing approximately $16.57 \%$ of the annual average of such loans of about 7865 million dinars during the second period, which illustrates the positive impact of the economic transformation on the value of agricultural loans long-term granted Agricultural Bank because the Libyan government has pumped funds for the Agricultural Bank to offset part of the years of economic and political blockade imposed on Libya and seek to achieve agricultural development through the implementation of agricultural projects, long-term funds that have been borrowed from the Agricultural Bank.

With regard to knowledge of the effects of these policies on the development of the total agricultural loans granted by the Agricultural Bank

Fayoum J. Agric. Res. \& Dev., Vol. 27, No.1, January, 2013 
shows that those loans in the case of a continuous increase significantly statistically for the first period were estimated at about 733.5 million dinars, representing about $6.6 \%$ of the annual average of such loans of about 11.1 million dinars during the first period. Increased to about 2.8968 billion dinars, representing about $11.77 \%$ of the annual average of such loans of about 24.62 million dinars during the second period, which demonstrates the positive impact of a period of economic transformation on the total agricultural loans granted by the Agricultural Bank which would have a positive impact on the agricultural projects that serve and achieve the objectives of agricultural development for the advancement of the agricultural sector to make it one of the leading sectors in the Libyan economy.

Review the values of rural credit granted by the Rural Bank in the field of agriculture amounted to about 4.05 million Libyan Dinar during the period 2003-2010 and the average loan granted by Rural Bank in the field of animal nearly 22.8 million dinars Libby during the same previous period, while the average value of loans maritime domain about 765 thousand Libyan dinars, while the average value of loans in the industrial area of about 5.6 million Libyan dinars during the previous period, and about 11.9 million Libyan dinars, nearly 33.4 million Libyan dinars in the literal field and the service during the previous period, respectively.

In the light of what illustrated by the results it recommends the following :

- Work to increase the amount of agricultural investment to agriculture even be able to achieve the highest possible efficiency of utilization of available genie has.

- The need to encourage investment in agricultural projects in order to play a leading role in agricultural development.

- It is too important to provide the producer by confessional agricultural loans because of their active role in agricultural development. 